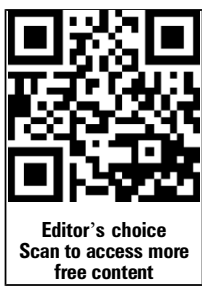

Scan to access m
free content

- Additional material is published online only. To view please visit the journal online (http://dx.doi.org/10.1136/ oemed-2014-102708).

${ }^{1}$ Provikmo Occupational Health Services, Bruges, Belgium ${ }^{2}$ Louvain centre for Toxicology and Applied Pharmacology (LTAP), Institut de Recherche Expérimentale et Clinique, Université Catholique de Louvain, Brussels, Belgium ${ }^{3}$ Environment and Health, Department of Public Health and Primary Care, KU Leuven, Leuven, Belgium

\section{Correspondence to} Dr Tom Geens, Provikmo Occupational Health Services, Dirk Martensstraat 26, Bruges B-8200, Belgium; tom.geens@provikmo.be

TG and EA contributed equally.

Received 17 November 2014 Revised 28 June 2015 Accepted 13 July 2015 Published Online First 6 August 2015

\title{
Exposure of hairdressers to aromatic diamines: an interventional study confirming the protective effect of adequate glove use
}

\author{
Tom Geens, ${ }^{1}$ Evelyne Aerts, ${ }^{1}$ Marc Borguet, ${ }^{1}$ Vincent Haufroid, ${ }^{2}$ Lode Godderis ${ }^{3}$
}

ABSTRACT
Objectives Many hairdressers leave their profession due to health problems, including occupational hand eczema, which has been associated with skin exposure to sensitising hair dye components such as paraphenylenediamine (PPD) and paratoluenediamine (PTD). Since the use of protective gloves is advised but without the short-term effect being known, our main goal was to attribute a significant biomarker reduction to adequate glove use, in a real work situation.

Methods 11 hairdressers were studied over 2 weeks. In the first week, they worked as usual and (re)used their gloves. Thereafter, we intervened to improve glove use during the second week. In both weeks, workplace exposure data were collected through observations, and systemic exposure was quantified by biomonitoring of PPD and PTD. The effect of improved glove use and other exposure determinants was studied through mixed models analysis.

Results We showed that improved glove use significantly reduced mean PTD concentrations from 24.1 before to $4.2 \mu \mathrm{g} / \mathrm{g}$ creatinine after the intervention ( $n=11$, third day postshift). In addition, mean PTD concentrations increased during the first week (14 times elevated after three consecutive shifts), but not during the second week. For PPD, no effect of improved glove use and no accumulation effect were detected.

Conclusions Our study is the first to deliver evidence for a significant reduction in systemic exposure to PTD through improved glove use. Disposable gloves should never be reused. PTD biomonitoring is shown to be a practical tool to quantify recent dermal exposure to oxidative hair dye components.

\section{INTRODUCTION}

Throughout history and across cultures, people have felt the need to change the natural colour of their skin, lips and hair. The use of Henna, for example, containing the natural dye 2-hydroxy1,4-naphtoquinone, can be traced back at least 4000 years. $^{1}$ Nowadays, in Europe, more than $60 \%$ of women and $5-10 \%$ of men between 30 and 60 years dye their hair on average 6-8 times per year. $^{2}$

In Europe, more than one million people (on average $0.6-0.7 \%$ of the working population per country) across more than 400000 hairdressing salons, reaching a potential of about 350 million customers, are professionally exposed. ${ }^{3} 4$ The sector is characterised by a young workforce (about $80 \%$ is

\section{What this paper adds}

- Paraphenylenediamine (PPD) and paratoluenediamine (PTD) are in the top 10 contact allergens in oxidative hair dyes but are also the economically most important hair dye precursor molecules.

- Hairdressers' exposures are high enough to constitute a risk of sensitisation and/or elicitation of allergic contact dermatitis, which is an important reason for leaving the profession.

- Previous studies could not find a significant effect of glove use on PPD or PTD dermal loadings or biomarker concentrations, nor could they demonstrate biomarker accumulation effects during the work week in the absence of adequate hand protection.

- We observed 11 hairdressers during two subsequent weeks: a first week with 'normal' glove use (eg, turned inside out and reuse) and, after intervention, a second week with adequate use (eg, use disposable gloves only once).

- Ours is the first study to demonstrate the protective effect of adequate glove use in a real work situation through a very practical intervention: in the second week the PTD biomarker does not accumulate and the overall PTD systemic exposure is much lower compared to the first week.

under 26 years of age) of predominantly part-time female (60-90\%) workers, often employed in owner-operated or micro-establishments $\quad(<10$ workers). ${ }^{4}$

Hairdressers and hairdressing apprentices belong to a high-risk population. They work many hours a day (including weekends), often in poor work postures, performing repetitive tasks in a stressful environment. Moreover, they are highly exposed to allergens (hair dye components, metals, perfumes, preservatives, etc) as well as to skin irritants (wet work, detergents, gloves, solvents, hydrogen peroxide, etc), in their professional and personal life. ${ }^{56}$ The combination of exposure to contact allergens and to skin irritants is a major risk factor for the development of occupational hand eczema (OHE), which has been shown previously to be an 
important reason to leave the profession or, even earlier, the training. ${ }^{7-10}$ While the 1 -year prevalence of hand eczema in the general population has been estimated as $8-11.8 \%$, OHE was shown to be $18-20 \%$ in trained hairdressers, and even up to $37 \%$ in apprentices. ${ }^{11-13}$

OHE represents $90 \%$ of all occupational skin diseases (OSD). The gross average OSD incidence in Europe has been estimated from national registries of notified occupational diseases at 7-15/10 000 workers per year, but for hairdressers, estimates of 110 (Denmark) and 240 (Bavaria) per 10000 workers per year have been made. ${ }^{14}$ Within Danish hairdressers diagnosed with OHE, approximately 50\% had irritant contact dermatitis, 25\% allergic contact dermatitis and the remaining 25\% a combination of both. ${ }^{15}$

The oxidative (or permanent) hair dyes represent $70-80 \%$ of the market in Europe. Other than in direct (temporary or semipermanent) and natural dyeing procedures, the colour is produced directly on and in the hair, assuring a resistance to almost any number of shampoos. The important ingredients of a permanent dye reaction mixture include an alkalinising agent such as ammonia, which opens the hair cuticle to assure deep penetration into the hair, an oxidising agent (almost exclusively hydrogen peroxide) to initiate the oxidation process of precursor molecules, the colourless precursors (called primary intermediates such as the arylamines paraphenylenediamine (PPD) or paratoluenediamine (PTD), p-aminophenols, pyrimidines and 4,5-diaminopyrazole) and, finally, coupler or modifier molecules (such as the metasubstituted aromatic derivate resorcinol, and pyridines and naphthols), which are chemically coupled to the oxidised precursors and responsible for the final colour achieved. ${ }^{1}$ The exact percentage of primary intermediates varies among the shades: darker colours contain a higher percentage than lighter ones. ${ }^{16}{ }^{17}$ The economically most important primary intermediates are PPD and PTD or 2,5-TDA. Cosmetics Europe estimated their worldwide use in 2005 at 150-200 metric tonnes a year. ${ }^{1}$ Both substances are of major importance in the sector, and losing approval to use them would create major problems for the hair dyeing industry. ${ }^{18}$

Considering the risks associated with these substances, their use is strictly regulated through the European Cosmetics Directive, which limits the final concentration in the applied hair colouring mixture to $2 \%$ for PPD and 4\% for PTD. ${ }^{19}$

In three recent consumer exposure studies, PPD and PTD are in the top 10 of contact allergens in oxidative hair dyes. ${ }^{20-22}$ Thus, patch testing with basic hair dye substances such as PPD, PTD, resorcinol and m-aminophenol and p-aminophenol (European baseline and hairdressers series), remains a common and justified tool in the diagnosis of OHE. ${ }^{23}$

In the prevention of OHE in hairdressers, it is essential to keep the exposure to allergens and irritants as low as possible. The abundant use of PPD and PTD as primary intermediates makes them important targets for monitoring occupational exposure to permanent hair dyes. Since concentrations of PPD and PTD in air were found to be inferior to the detection limits of the methods $\left(0.1-1.0 \mu \mathrm{g} / \mathrm{m}^{3}\right)$, inhalation is not considered to be an important route of exposure. ${ }^{24} 25$ In contrast, mean dermal loads (amounts adhered to the skin) of 153-454 nmol PPD/hand (17-49 $\mu \mathrm{g})$ and 71-192 nmol PTD/hand (9-24 $\mu \mathrm{g})$, depending on the moment of sampling, have been demonstrated; quantities large enough to constitute a risk of sensitisation and/or elicitation of allergic contact dermatitis. ${ }^{26}$

According to cosmetic manufacturers, dermal uptake (amounts penetrating through intact skin) can be avoided using suitable gloves. Permeation of hair dye ingredients through different types of protective gloves has been studied and investigators concluded that, if used following the instructions of the manufacturer, all tested gloves gave considerable protection. ${ }^{27} 28$ Unfortunately, this could not yet be confirmed in real work situations. $^{29} 30$ Lind et al could not find a reducing effect of glove use on dermal loads of PPD and PTD. Similarly, Gube et al did not observe an influence of glove use on systemic exposure of hairdressers to PPD and PTD, nor could they show differences in systemic exposure during the workweek, which were to be expected considering a half-life of $8 \mathrm{~h}$ for PTD and 8-12 h for PPD. Explanations were sought in, for example, reporting bias, and glove reuse and misuse, and even for the absence of a protective affect. ${ }^{29} 3132$ In contrast, glove use has been shown to be effective in reducing the incidence of $\mathrm{OHE}$ in hairdressing apprentices. ${ }^{33}$

Our main purpose was to assess whether exposure of hairdressers to aromatic diamines, using PPD and PTD as biomarkers of exposure, could be reduced by adequate use of gloves: for each customer a new glove pair, used throughout the whole dyeing process. In addition, this study aimed at demonstrating accumulation of aromatic diamines in hairdressers across the work week, when gloves are for example, incorrectly reused.

Evidence would provide occupational hygienists and physicians with a very powerful tool to quantify the exposure to hair dye ingredients in different real work situations.

\section{METHODS}

\section{Participants}

The target was to recruit a maximum of 20 hairdressers, employed in two or more hairdressing salons with at least four employees. Salons were filtered from the Provikmo employer database (Provikmo Occupational Health Services, Bruges, Belgium). Ten salons were visited to present the study aims. Our inclusion criteria were: participants working at least three representative and consecutive days in the salon, after at least two consecutive days without exercising hairdressing activities (in the salon or elsewhere), and agreeing to and signing the informed consent. Our exclusion criteria were: participants who had coloured their own hair less than 7 days before the start of the study or were planning to do this during the study, and participants who had performed any hair colouring activity in the $48 \mathrm{~h}$ prior to the start of the study or who were planning to do this after working hours in the salon during the study. Finally, participants exposed to Henna (through tattoos or natural hair dyes) the last month before or during the study were also to be excluded.

\section{Study design}

We carried out a non-randomised uncontrolled before-and-after study. The field experiment took place over the course of 2 weeks in each participating salon. Each subject was followed over both weeks. The first measurement moment of each week indicates the background biomarker levels. In the first week, hairdressers (re)used gloves as usual. Exposure to oxidative dyes was monitored through continuous observation by a single researcher recording the handlings of the exposed workers and collecting preshift and postshift urine samples in three subsequent days with exposure, after at least 2 days without exposure.

The same observation and sampling strategy was followed in the second week, after intervention.

\section{Intervention}

Before the intervention, single-sized powdered latex gloves with short cuffs were available to the hairdressers in both salons. They were allowed to use and reuse them. The reuse process 
was observed and documented (figure 1). At the end of the first week, oral and written instructions on correct glove use were provided. Powder-free nitrile gloves with a $300 \mathrm{~mm}$ long cuff (Touch'n Tuff 92-605, Ansell, Iselin, New Jersey, USA) were provided in four sizes (small, medium, large and extra-large), assuring good fit at the wrist. During the second week, the use was observed and corrected when necessary. Reuse was never allowed. Use was enforced during the entire dyeing procedure except during cutting and drying (see online supplementary table). Before and after intervention, the same work clothes (three quarter sleeves) and work material were used.

\section{Hair dyeing procedure and observation of exposure}

Participants were observed in both weeks of the study, in order to document all handlings associated with possible dermal exposure to aromatic diamines during the dyeing activities. This included mixing permanent dye (first step), application of dye (whole hair and single strands) and cleaning of the application tools (second step), washing the hair after dyeing (third step), and cutting and drying of coloured hair (fourth step). Numbers and duration of each of these steps were recorded per hairdresser for three subsequent working days.

The hair dye products Majirel, Inoa, Inoa Suprême and Diarichesse (L'Oréal, Clichy, France) were used during the study. Exact PPD and PTD concentrations were, unfortunately, not provided by the manufacturer. Colour identification numbers of the hair dye shades as well as the processed volumes and the usage times per product were recorded. Given the large amount of possible shades for the products applied, the 10-point scale of the manufacturer was categorised into three colour groups. Black, brown black and darkest brown (shades 1-3) were recorded as dark colours; dark brown, brown and light brown (shades 4-6) as middle colours; and dark, blonde, light blonde and very light blonde (shades 7-10) represented light colours.

From the observation of colour categories and used volumes, we estimated the daily manipulated PPD and PTD volumes per person, assuming that the content of primary intermediates in light shades is around $0.05 \%$ and around $1.50 \%$ in dark shades. ${ }^{17}$ We estimated the content in our middle class at $0.27 \%$ (assuming that the concentration rises exponentially). These additional exposures describing parameters PPDexp and PTDexp $(\mathrm{mL})$ were added to the data set.

\section{Biomonitoring of PTD and PPD}

Systemic exposure to permanent hair dyes was measured by means of biomonitoring of the aromatic diamines PTD and PPD in urine.

Urine samples were collected in polypropylene recipient cups preshift and postshift each day of the study. Hairdressers were instructed to wash their hands carefully before providing urine samples to avoid external contamination. Immediately after
Figure 1 Glove reuse as observed before intervention. Gloves were washed (A), patted dry (B) and dusted with talcum powder ( $C$ and $D)$. The gloves were then peeled off (E) so that the outer contaminated surface became the inside of the glove when it was reused. The effect of the contamination with hair dye is dramatic ( $F$, right) in comparison with a new glove $(F$, left)
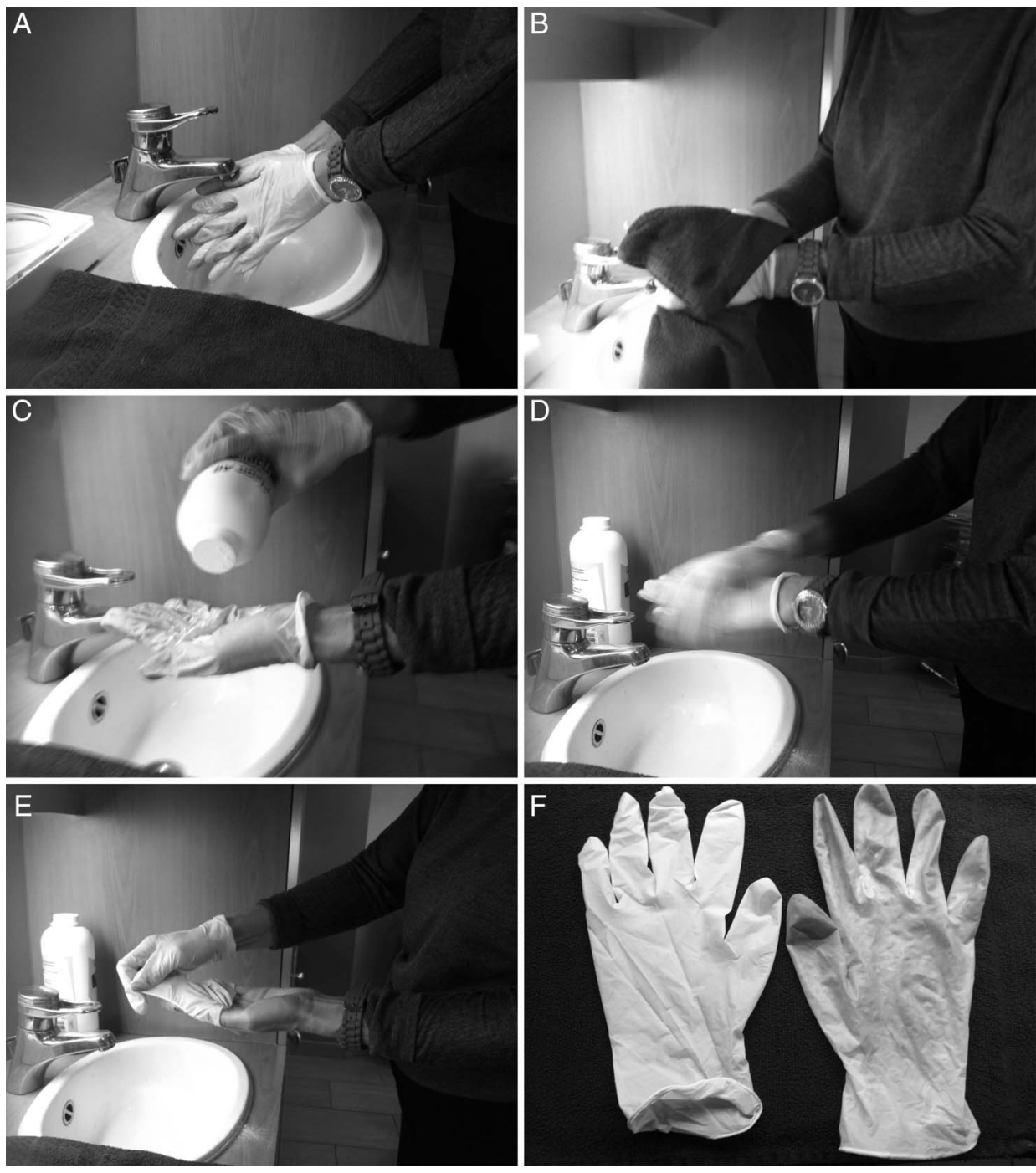
collection, $10 \mathrm{~mL}$ urine was transferred into a new polypropylene cup containing $0.5 \mathrm{~mL} \mathrm{HCl} 6 \mathrm{M}$. A second aliquot of $5 \mathrm{~mL}$ was transferred to a second, smaller recipient (without acid) for measurement of creatinine. Samples were immediately stored at $4^{\circ} \mathrm{C}$ and frozen at $-18^{\circ} \mathrm{C}$ within $24 \mathrm{~h}$.

For each participating hairdresser, preshift and postshift samples of the first and third workday from both study weeks were analysed. Analysis of PTD and PPD was performed using gas chromatography with mass spectrometry in the Laboratory of Industrial and Environmental Toxicology (Université Catholique de Louvain, Brussels, Belgium), as described earlier. ${ }^{34}$ For both analytes, the limit of detection (LOD) and limit of quantification (LOQ) were 0.1 and $0.5 \mu \mathrm{g} / \mathrm{L}$, respectively.

\section{Statistics}

Results of the observations were registered in MS Excel 2010 (Microsoft, Redmond, Washington, USA). The analytical results of PTD, PPD $(\mu \mathrm{g} / \mathrm{L})$ and creatinine $(\mathrm{g} / \mathrm{L})$ were provided by the laboratory in an MS Excel 2010 file. Creatinine normalised concentrations were calculated by dividing each analytical result with its creatinine content. Both files were merged into one IBM SPSS Statistics 20 data file (IBM Corporation, Somers, New York, USA) for subsequent statistical analyses. Differences in external exposure (duration and frequency of operations with exposure to aromatic diamines) between study days and study weeks were examined with the independent samples Kruskal-Wallis test. Statistical significance was set at $\mathrm{p}<0.05$ (two tailed).

PTD and PPD concentrations (in both $\mu \mathrm{g} / \mathrm{L}$ and $\mu \mathrm{g} / \mathrm{g}$ creatinine) showed skewed distributions, requiring the data to be In-transformed prior to analysis. Individual hairdresser exposures during the work week were visually presented using profile plots displaying $\operatorname{lnPTD}$ and $\operatorname{lnPPD}$ in time.

Determinants of systemic exposure to PTD and PPD (transformed $\mu \mathrm{g} / \mathrm{g}$ creatinine) were examined with linear mixed models analyses (MIXED procedure in SPSS). Hairdresser was considered as random effect and week as the primary fixed effect of interest; manipulated volume, exposure times (the four process steps and three colour types), countings (process steps and colour types) and the additional exposure describing parameters PPDexp and PTDexp (transformed $\mathrm{mL}$ ) were considered as level one fixed effects, and salon, sex, smoking, alcohol and medication as level two fixed effects.

Variances were estimated as between worker (across participants) and within worker (repeated measurements) variance components. Significance of variance components was investigated using restricted maximum likelihood estimates used for likelihood ratio testing for covariance parameters.

A step-up model building strategy was followed. ${ }^{35}$ In the first step, a naïve model (ignoring the repeats within the participants) was compared with a random intercept and/or slope model (accounting for the repeats). In the second step, level one covariates were introduced and backward removed (highest $p>0.05$ was removed first, iteration continued until all $\mathrm{p}<0.05$ ). In the third step, the primary fixed effect of interest was added, and in the fourth and final step, level two covariates were introduced and backward removed (same criteria).

\section{RESULTS}

\section{Participants}

Eleven hairdressers from two typical Flemish microestablishments (two male employers and four and five of their female employees, respectively) took part in the present study, conducted during January 31-February 11 (salon 1) and February 28-March 10 (salon 2), 2012. The age of the participants ranged from 18-49 years, with a mean of 31 years. None of them had to be excluded during the study.

\section{The intervention strongly altered the glove use}

In week 1 , hairdressers were wearing gloves only while dyeing whole hair and rinsing dark (and in some cases also middle) colours. Gloves were reused. This process was carefully observed and is documented in figure 1 . In week 2 , gloves were used in all steps, except during cutting and drying. Gloves were never reused. Differences in glove use before and after the intervention are summarised in an online supplementary table.

\section{Comparable duration and frequency of process steps} and product use before and after intervention

Mean durations of handlings across the 11 participants are displayed in table 1. Mean length of the workday was comparable in both weeks. Length of exposure during the different process steps and to different colour types, was comparable within and between both weeks. Kruskall-Wallis tests between weeks $(\mathrm{df}=1)$, between days within week $(\mathrm{df}=2)$ and between days across weeks $(\mathrm{df}=5)$ did not reveal any significant difference (all $p>0.157$ ), nor did the results for the frequencies (number of process steps) and processed volumes (three colour types and the exposure summarising parameters PPDexp and PTDexp) (data not shown).

\section{Biomarker descriptive statistics at the various sampling moments}

A total of 88 urinary samples were analysed (four samples before and after intervention for each of the 11 participants). Descriptive statistics are shown for creatinine-normalised PTD and PPD biomarkers before (week 1) and after intervention (week 2) in table 1. For PTD, geometric mean, arithmetic mean and median are higher in subsequent measurement moments in week 1, but not in week 2. For PPD, this is not observed. The maximum PTD value of $154.5 \mu \mathrm{g} / \mathrm{g}$ creatinine week 1 , day 3, was an outlier, but not an extreme value.

Profile plots of the individual hairdressers' PTD (left) and PPD (right) biomarker concentrations are shown in an online supplementary figure. At the first measurement moment, results from week 1 (dashed line) and week 2 (solid line) overlap for both biomarkers. At the last measurement moment, dashed lines end up generally higher than the solid lines for PTD, suggesting a biomarker accumulation effect in week 1 , but not in week 2 . For PPD, this effect is not observed. The overall PPD lines show less variation in time (see online supplementary figure).

\section{Determinants of systemic exposure}

In our final model, a significant variance in intercepts between hairdressers' biomarker concentration (quantified through the ln of the measured PTD concentration in $\mu \mathrm{g} / \mathrm{g}$ creatinine) $\left(\sigma_{\beta}^{2}=0.31\right)$ is present after correcting for exposure to PTD (quantified through the $\ln$ of the parameter PTDexp, calculated from the observations). Only a random intercept was retained. The biomarker concentration increased linearly with increasing exposure levels $(p=0.01)$ : with every unit increase in $\ln$ PTDexp, $\operatorname{lnPTD}$ increased by 0.57 . The residual variance attributed to the repeats (within subject variance or $\sigma_{\varepsilon}^{2}$ ) was 0.42 . The primary fixed effect of interest was highly significant $(\mathrm{p}<0.001)$ : after intervention, the $\ln$ PTD was on average 0.96 lower than before. All other level 1 or level 2 covariates were removed since they 
Table 1 Comparison of mean duration of exposure between work days and weeks

\begin{tabular}{|c|c|c|c|c|c|c|c|c|c|c|c|}
\hline \multirow[b]{2}{*}{ Week } & \multirow[b]{2}{*}{ Day } & \multirow{2}{*}{$\begin{array}{l}\text { Time } \\
\text { work day }\end{array}$} & \multirow{2}{*}{$\begin{array}{l}\text { Time without } \\
\text { exposure }\end{array}$} & \multirow{2}{*}{$\begin{array}{l}\text { Time with } \\
\text { exposure }\end{array}$} & \multicolumn{4}{|c|}{ Time with exposure during } & \multicolumn{3}{|c|}{ Time exposed to } \\
\hline & & & & & Step 1 & Step 2 & Step 3 & Step 4 & Light colours & Middle colours & Dark colours \\
\hline \multirow[t]{4}{*}{ Week 1} & 1 & 9:54 & 8:08 & 1:46 & $0: 08$ & $0: 33$ & $0: 21$ & $0: 42$ & $0: 41$ & $0: 59$ & $0: 04$ \\
\hline & 2 & $10: 04$ & $8: 18$ & 1:46 & $0: 08$ & $0: 38$ & $0: 20$ & $0: 37$ & $0: 46$ & $0: 52$ & 0:06 \\
\hline & 3 & $10: 27$ & $8: 42$ & $1: 45$ & $0: 05$ & $0: 26$ & $0: 21$ & $0: 50$ & $0: 43$ & $0: 54$ & $0: 06$ \\
\hline & $1-3^{*}$ & $10: 08$ & $8: 22$ & 1:45 & $0: 07$ & $0: 32$ & $0: 20$ & $0: 43$ & $0: 43$ & $0: 55$ & $0: 05$ \\
\hline \multirow[t]{4}{*}{ Week 2} & 1 & 10:04 & $8: 12$ & 1:52 & $0: 07$ & $0: 33$ & $0: 24$ & $0: 46$ & $0: 56$ & $0: 47$ & 0:08 \\
\hline & 2 & 10:06 & 7:40 & $2: 26$ & $0: 10$ & $0: 51$ & $0: 28$ & $0: 54$ & $0: 56$ & $1: 20$ & 0:09 \\
\hline & 3 & 10:16 & $8: 15$ & 2:01 & $0: 08$ & $0: 34$ & $0: 21$ & $0: 56$ & $0: 42$ & $1: 15$ & $0: 02$ \\
\hline & $1-3^{*}$ & 10:08 & 8:02 & 2:06 & $0: 08$ & $0: 39$ & $0: 24$ & $0: 52$ & $0: 51$ & 1:07 & $0: 06$ \\
\hline
\end{tabular}

*Times in italic are mean times for three consecutive days.

did not have any significant effect. For PPD, exposure (lnPPDexp) and intervention were not found to have any significant effect in predicting the biomarker level (lnPPD). The final PTD model and the corresponding PPD model are summarised in table 2. Excluding the PTD value of $154.5 \mu \mathrm{g} / \mathrm{g}$ creatinine week 1 day 3 did not affect the conclusions.

\section{Accumulation of PTD before, but not after, the intervention}

The same mixed models approach was used to investigate an accumulation effect during the week before and after intervention. Since the intervention did not have any effect on PPD biomarker concentration, this was only carried out for PTD. The internal exposure preshift and postshift day 1 is significantly lower than the reference level reached day 3 postshift in week 1 , but not in week 2 (table 3). Excluding the PTD value of $154.5 \mu \mathrm{g} / \mathrm{g}$ creatinine week 1 , day 3 , did not affect the conclusions.

\section{DISCUSSION}

In the present study, we prove that systemic exposure of hairdressers to aromatic diamines is reduced by adequate glove use. Our results indicate that exposure to PTD, but not PPD, is lower after intervention (tables 2 and 3). The presented linear mixed models account for fluctuations in exposure in time, which were not found to be significant across participants (table 1). The trend of increasing PTD levels towards the end of a 3-day working period was apparent before, but not after, intervention (table 3 and see online supplementary figure), which supports the expected accumulation effect. For PPD, however, no such pattern was observed (see online supplementary figure).
Correct glove use is a good preventive measure to protect hairdressers from the development of OHE. ${ }^{33}$ Laboratory studies have shown that all glove types provide sufficient protection for at least $30 \mathrm{~min}$ and that glove use reduces the incidence of OHE. ${ }^{27} 28{ }^{33}$ However, previous studies could not prove an immediate influence of glove use on dermal loading or systemic exposure of hairdressers to hair dye compounds in real work situations. $^{2629}$

Lind et $a l^{26}$ assessed skin exposure of hairdressers to PPD, PTD and resorcinol. Their analysis of hand rinse sampling results showed that glove use did not influence dermal loading and hypothesised that this was due to improper glove use: reusing disposable latex gloves for 2-3 months or until these were damaged or torn. Gube et $a l^{29}$ quantified urinary excretion of PPD and PTD in hairdressers and concluded that there was no significant effect of (self-reported) glove use on systemic exposure, possibly due to misreporting. Our study confirms improper reuse (figure 1) and corrects it, deals with misreporting through detailed observation (table 1 , see online supplementary table) and refutes any doubt on the protective effect (tables 2-4). ${ }^{26} 36$

As both Lind et $a l^{30}$ and Gube et $a l^{29}$ relied on interviews and self-reporting, an unknown bias was introduced, which made a significant protective effect of glove use hard to investigate. This was overcome in our study through strict and detailed observation by a single researcher, thus avoiding reporter and observer bias.

Since the intervention in our study only corrects the improper glove use, and not the exposure pattern (table 1 and see online supplementary table), we prove that correct glove use helps to reduce systemic exposure in a real work situation (table 2).

Table 2 Parameter estimates for the final mixed effect models on In transformed biomarker concentrations

\begin{tabular}{|c|c|c|c|c|}
\hline \multirow{2}{*}{$\begin{array}{l}\text { Model } \\
\text { Parameter }\end{array}$} & \multicolumn{2}{|l|}{$\operatorname{lnPTD}(n=66)^{*}$} & \multicolumn{2}{|l|}{$\ln P P D(n=66)^{*}$} \\
\hline & $\boldsymbol{\beta}$ & $\mathrm{p}$ Value & $\beta$ & p Value \\
\hline Intercept $\left(\beta_{0}\right)$ & $-0.066(-1.011$ to 0.879$)$ & 0.889 & $-0.084(-0.880$ to 0.713$)$ & 0.834 \\
\hline \multicolumn{5}{|l|}{ Week $\left(\beta_{1}\right)$} \\
\hline 1 & 0.963 (0.641 to 1.285$)$ & $<0.001$ & $-0.093(-0.507$ to 0.322$)$ & 0.655 \\
\hline 2 & $0+$ & & $0 \dagger$ & \\
\hline Exposure $\neq\left(\beta_{2}\right)$ & 0.565 (0.142 to 0.989$)$ & 0.010 & $0.126(-0.386$ to 0.638$)$ & 0.624 \\
\hline Random intercept $\left(\sigma_{\beta}^{2}\right)$ & 0.306 (0.106 to 0.884$)$ & & 0.229 (0.062 to 0.853$)$ & \\
\hline Measurement error $\left(\sigma_{\varepsilon}^{2}\right)$ & 0.421 (0.289 to 0.612$)$ & & $0.698(0.480$ to 1.017$)$ & \\
\hline
\end{tabular}

${ }^{*} \mathrm{n}$, number of samples; since the primary fixed effect of interest was the difference between Week 1 and Week 2, the baseline samples Day 1 preshift ( $\mathrm{n}=11$ ) were excluded from the analysis, as no effect of any kind of intervention can be expected at a first measurement moment after 2 days without exposure in both weeks.

tThe last category is used as reference level, estimates are given with their corresponding $95 \% \mathrm{Cl}$ (LCL to UCL).

¥The exposure parameters used were InPTDexp for the InPTD model and InPPDexp for the InPPD model.

PPD, paraphenylenediamine; PTD, paratoluenediamine. 
Table 3 Parameter estimates for the final mixed effect model on In transformed PTD concentrations during both weeks of the study

\begin{tabular}{|c|c|c|c|c|}
\hline \multirow{2}{*}{$\begin{array}{l}\text { Model } \\
\text { Parameter }\end{array}$} & \multicolumn{2}{|l|}{ Week $1(n=44)^{*}$} & \multicolumn{2}{|l|}{ Week $2(n=44)^{*}$} \\
\hline & $\boldsymbol{\beta}$ & $\mathrm{p}$ Value & $\boldsymbol{\beta}$ & $\mathrm{p}$ Value \\
\hline Intercept $\left(\beta_{0}\right)$ & $1.353(0.324$ to 2.382$)$ & 0.011 & $0.394(-0.911$ to 1.699$)$ & 0.543 \\
\hline \multicolumn{5}{|l|}{ Time $\left(\beta_{1}\right)$} \\
\hline Day 1 preshift $(0 \mathrm{~h})$ & $-2.230(-2.748$ to -1.713$)$ & $<0.001$ & $-0.357(-0.848$ to 0.134$)$ & 0.149 \\
\hline Day 1 postshift $(10 \mathrm{~h})$ & $-0.877(-1.394$ to -0.360$)$ & 0.002 & $-0.195(-0.687$ to 0.296$)$ & 0.424 \\
\hline Day 3 preshift (48 h) & $-0.375(-0.889$ to 0.139$)$ & 0.147 & $0.059(-0.431$ to 0.550$)$ & 0.807 \\
\hline Day 3 postshift (58 h) & $0+$ & & $0+$ & \\
\hline $\operatorname{lnPTDexp}\left(\beta_{2}\right)$ & 0.546 (0.116 to 0.976$)$ & 0.014 & $0.359(-0.251$ to 0.969$)$ & 0.239 \\
\hline Random intercept $\left(\sigma_{\beta}^{2}\right)$ & 0.226 (0.066 to 0.776$)$ & & $0.243(0.079$ to 0.751$)$ & \\
\hline Measurement error $\left(\sigma_{\varepsilon}^{2}\right)$ & $0.350(0.214$ to 0.570$)$ & & $0.320(0.197$ to 0.519$)$ & \\
\hline
\end{tabular}

${ }^{*} n$, number of samples; since the model is constructed separately for both weeks, only half of the data is used in each model.

tThe last category is used as reference level, estimates are given with their corresponding $95 \% \mathrm{Cl}$ (LCL to UCL).

PTD, paratoluenediamine.

Considering a previously calculated half-life of $8 \mathrm{~h}$ for PTD and $8-12 \mathrm{~h}$ for PPD, ${ }^{29} 3132$ we expected, as did others, but were the first to prove, differences in systemic exposure to PTD across the work week. Analysis of our results showed that PTD accumulated before intervention. The absence hereof after intervention can only be attributed to the improved glove use.

Gube et $a l^{29}$ detected neither an intrashift effect, nor an effect during the workweek, which they attributed to overall quite heterogeneous exposure among hairdressers within and across work shifts. A direct comparison of the results of both studies is difficult due to the many differences (hair colouring products, PPD and PTD content, work circumstances, methodological approach to describe exposure and glove use, exclusion and inclusion criteria, analytical sample analysis, statistical parameters and tests to describe and interpret the results). ${ }^{29}$

Comparing the PTD medians (day 1 preshift, day 3 preshift and postshift), our results are generally six times higher compared to Gube et al (table 4), and for PPD, 33\% (29/88) of our results were $<\operatorname{LOQ}(0.5 \mu \mathrm{g} / \mathrm{L})$ (data not presented), while Gube et $a^{29}$ found only $8 \%(4 / 52)$ samples $>$ LOQ $(1 \mu \mathrm{g} / \mathrm{L})$.

Despite this difference, there is also a remarkable similarity between both studies: PTD (but not PPD) median concentrations day 3 preshift and postshift are sixfold to sevenfold higher, compared to day 1 preshift (table 2). ${ }^{29}$

The most important strength of the present study is its repeated measures design following the same participants before and after intervention, and the fact that exposure was characterised in detail, following rigorous observation by a single researcher. The participants were reasonably young, predominantly female workers employed in small salons, which makes our study population a typical sample. ${ }^{4}$ The intended level of 20 participants was not reached, but even with only 11 hairdressers, there was enough evidence to reach the study aims.

Since hairdressers are in general part-time workers with personal work schedules within their salon, a follow up-period of

Table 4 PTD and PPD ( $\mu \mathrm{g} / \mathrm{g}$ creatinine) descriptive statistics for the eleven hairdressers at various sampling times

\begin{tabular}{|c|c|c|c|c|c|c|c|c|}
\hline \multirow{2}{*}{$\begin{array}{l}\text { Parameter } \\
\text { Measurement* }\end{array}$} & \multicolumn{4}{|l|}{ PTD } & \multicolumn{4}{|l|}{ PPD } \\
\hline & 0 & 1 & 2 & 3 & 0 & 1 & 2 & 3 \\
\hline \multicolumn{9}{|l|}{ Week 1} \\
\hline $\mathrm{n}$ & 11 & 11 & 11 & 11 & 11 & 11 & 11 & 11 \\
\hline GM & 1.24 & 4.80 & 8.56 & 12.45 & 1.01 & 0.95 & 0.86 & 1.23 \\
\hline GS & 2.43 & 1.89 & 1.92 & 2.67 & 2.92 & 2.50 & 1.69 & 2.29 \\
\hline $\mathrm{AM}$ & 1.74 & 5.87 & 10.30 & 24.08 & 2.18 & 1.63 & 0.97 & 1.78 \\
\hline AS & 1.47 & 4.67 & 6.27 & 43.63 & 4.20 & 2.59 & 0.54 & 1.92 \\
\hline Min & 0.31 & 2.03 & 3.50 & 4.26 & 0.33 & 0.29 & 0.40 & 0.42 \\
\hline Med & 1.44 & 5.34 & 7.54 & 8.67 & 0.85 & 0.99 & 0.83 & 1.13 \\
\hline Max & 5.07 & 19.00 & 20.21 & 154.50 & 14.71 & 9.33 & 2.00 & 6.40 \\
\hline \multicolumn{9}{|l|}{ Week 2} \\
\hline $\mathrm{n}$ & 11 & 11 & 11 & 11 & 11 & 11 & 11 & 11 \\
\hline GM & 2.16 & 2.53 & 3.23 & 3.04 & 1.07 & 0.92 & 1.02 & 1.37 \\
\hline GS & 1.36 & 1.84 & 2.88 & 2.66 & 3.29 & 2.99 & 2.70 & 3.99 \\
\hline$A M$ & 2.26 & 2.96 & 5.20 & 4.23 & 3.06 & 2.18 & 1.86 & 4.48 \\
\hline AS & 0.75 & 1.69 & 5.76 & 3.17 & 7.05 & 4.60 & 3.03 & 10.13 \\
\hline Min & 1.37 & 0.66 & 0.49 & 0.37 & 0.24 & 0.26 & 0.36 & 0.29 \\
\hline Med & 2.14 & 2.67 & 3.26 & 3.59 & 0.82 & 0.69 & 0.88 & 1.21 \\
\hline Max & 4.03 & 6.64 & 20.67 & 11.53 & 24.22 & 16.00 & 10.83 & 34.76 \\
\hline
\end{tabular}

*Measurement number; 0 , day 1 preshift; 1 , day 1 postshift; 2, day 3 preshift; 3 day 3 postshift.

AM, arithmetic mean; AS, arithmetic SD; GM, geometric mean; GS, geometric SD; Max, maximum; Min, minimum; Med, median; n, number of samples; PPD, paraphenylenediamine; PTD, paratoluenediamine. 
three subsequent days was the maximum we could achieve in our study population.

A weakness of our study is the lack of a control group to adjust for effects other than, for example, exposure. This was an explicit choice of the researchers to give all 11 hairdressers the chance to improve their dermal protection. Inclusion of a control group and random assignment of participants to it would have allowed to adjust also for learning effects, for example.

The absence of an effect of glove use on PPD systemic exposure (tables 2-4) is in concordance with what Gube et $a^{29}$ found. While in their study the replacement of PPD with PTD in Germany might explain a majority of results $<\mathrm{LOQ}$, this is not the case in our study. Of course, the PTD content is generally higher than the PPD content: the legal limit for PTD is twice as high and PTD is known to be $50 \%$ less efficient than PPD (double the quantities are needed to achieve the same effect), which explains previous findings of PPD and PTD contents of $0.004-0.250 \%$ and $0.019-0.447 \%$ in 22 hair dyeing mixtures, respectively. ${ }^{18} 1926$

But more important, and in contradiction to the hypothesis that PTD absorption and excretion may be similar to those of PPD, both substances may differ more than previously thought. ${ }^{29} 32$ After application of one unit of PTD on human skin in oxidative circumstances, not $0.5 \%$ as for PPD, but $3.5 \%$ to $4 \%$ of the initial dose, could be recovered in urine. $^{37} 38$ This is also what one would expect from permeation modelling: using the NIOSH Skin Permeation Calculator and lowering the maximum soluble quantity to the legal PPD (CAS 105-50-3) maximum concentration of $2 \%$, the models predict a flux range of $1,5-9 \mu \mathrm{g} / \mathrm{cm}^{2} / \mathrm{h} .{ }^{39}$ Using read-across with the same method for 2,4- (CAS 95-80-7) and 2,6-TDA (CAS 823-40-5) at a concentration of $4 \%$, a flux of 6-24 $\mu \mathrm{g} /$ $\mathrm{cm}^{2} / \mathrm{h}$ is expected. We believe that this would be a better explanation for PTD results ending up 4-8 times higher than PPD, in absence of adequate dermal protection. More research is needed to confirm this. The lack of variation in PPD results may also be due to the fact that many results in our study were $>$ LOD but $<$ LOQ.

We proved that adequate use of nitrile gloves significantly reduces systemic exposure of hairdressers to PTD. This is the first time a convincing protective influence of glove use on systemic exposure to a very common hair dye compound is shown in real workplace conditions. We conclude that PTD is a suitable target molecule to monitor recent exposure. Occupational hygienists and physicians should further explore the sense of PTD biomonitoring as a useful tool in short term exposure follow-up in workplace assessments.

The practical take home message is that proper training in the use of adequate gloves can be effective in reducing daily exposure in the short term. The biggest challenge, ensuring that the hairdressers implement this also in the long term, however, remains. A biomarker such as PTD can be useful in assessing this.

Protective gloves should be worn while mixing colour, application of colour and during rinsing and washing after dyeing.

Gloves need to fit properly and cuffs need to be long enough, in order to prevent rinsing water from leaking into them, and splattering of droplets of colour cream onto bare forearms.

Since gloves are inconvenient during hair cutting, cutting might precede the actual colouring procedure for further exposure reduction.

Because of the risk of contamination while reusing gloves, we confirm the findings of Lind $e t a l^{30}$ and strongly advise against reuse of disposable gloves.
Twitter Follow Tom Geens at @tgeens

Acknowledgements The authors wish to thank the employers and personnel of both hairdressing salons that participated in this study. Liesbeth Bruckers (CenStat, UHasselt, Hasselt University, Hasselt, Belgium) is gratefully acknowledged for her valuable statistical advice and her help in unravelling the SPSS mixed models procedure.

Contributors EA carried out the preceding literature study and prepared the study design, executed the field research and wrote the first draft of the present study paper; TG, LG and VH decided on the final study aims and required design to reach these. MB assisted in the discussion and interpretation of the study results and the writing of the final paper. TG and EA contributed equally.

Funding TG, EA and MB were financially supported by Provikmo, VH was financed by UCL, and LG by KULeuven, to conduct this study. The workers agreed to participate for free in the study.

Competing interests None declared.

Patient consent Obtained.

Ethics approval UZ Leuven Medical Ethics Committee, University of Leuven, Leuven, Belgium (ML7777)

Provenance and peer review Not commissioned; externally peer reviewed.

\section{REFERENCES}

1 Occupational exposures of hairdressers and barbers and personal use of hair colourants. IARC monographs on the evaluation of carcinogenic risks to humans. Some aromatic amines, organic dyes, and related exposures. Lyon, France: International Agency for Research on Cancer, 2010:499-658

2 Hair dye products-European Commission. European Commission, 2014

3 Eeckelaert L, Dontas S, Georgiadou E, et al. Occupational health and safety in the hairdressing sector. Luxembourg, Luxembourg: European Agency for Safety and Health at Work, 2014:1-30.

4 Weber T, Nevala A-M, Mantouvalou K. Study on social policy effects resulting from the scope of application of the European framework agreement on the prevention of health risks in the hairdressing sector. Birmingham, UK: GHK, 2011:1-112.

5 Hansen HS, Johansen JD, Thyssen JP, et al. Personal use of hair dyes and temporary black tattoos in Copenhagen hairdressers. Ann Occup Hyg 2010;54:453-8

6 Krecisz B, Kiec-Swierczynska M, Chomiczewska D. Dermatological screening and results of patch testing among Polish apprentice hairdressers. Contact Dermatitis 2011:64:90-5.

7 Leino $\mathrm{T}$, Tuomi $\mathrm{K}$, Paakkulainen $\mathrm{H}$, et al. Health reasons for leaving the profession as determined among Finnish hairdressers in 1980-1995. Int Arch Occup Environ Health 1999;72:56-9.

8 Lysdal SH, Sosted H, Andersen KE, et al. Hand eczema in hairdressers: a Danish register-based study of the prevalence of hand eczema and its career consequences. Contact Dermatitis 2011;65:151-8.

9 Uter W, Pfahlberg A, Gefeller 0, et al. Prevalence and incidence of hand dermatitis in hairdressing apprentices: results of the POSH study. Prevention of occupational skin disease in hairdressers. Int Arch Occup Environ Health 1998;71:487-92.

10 Uter W, Pfahlberg A, Gefeller O, et al. Hand dermatitis in a prospectively-followed cohort of hairdressing apprentices: final results of the POSH study. Prevention of occupational skin disease in hairdressers. Contact Dermatitis 1999;41:280-6.

11 Meding B. Epidemiology of hand eczema in an industrial city. Acta Derm Venereol Suppl (Stockh) 1990;153:1-43.

12 Meding B, Liden C, Berglind N. Self-diagnosed dermatitis in adults. Results from a population survey in Stockholm. Contact Dermatitis 2001;45:341-5.

13 Meding B, Jarvholm B. Incidence of hand eczema-a population-based retrospective study. J Invest Dermatol 2004;122:873-7.

14 Diepgen TL. Occupational skin-disease data in Europe. Int Arch Occup Environ Health 2003;76:331-8.

15 Skoet $\mathrm{R}$, Olsen J, Mathiesen B, et al. A survey of occupational hand eczema in Denmark. Contact Dermatitis 2004:51:159-66.

16 International Agency for Research on Cancer. IARC monographs of the evaluation of carcinogenic risks to humans. Some aromatic amines, organic dyes and related exposures. Vol 99. Lyon: World health organization, 2010.

17 Nohynek GJ, Fautz R, Benech-Kieffer F, et al. Toxicity and human health risk of hair dyes. Food Chem Toxicol 2004;42:517-43.

18 Corbett JF. An historical review of the use of dye precursors in the formulation of commercial oxidation hair dyes. Dyes Pigments 1999;41:127-36.

19 European Union. Council directive of 27 July 1976 on the approximation of the laws of the Member States relating to cosmetic products (76/768/EEC). Official Journal of the European Union 1976;L262:169-200.

20 Hamann D, Yazar K, Hamann CR, et al. p-Phenylenediamine and other allergens in hair dye products in the United States: a consumer exposure study. Contact Dermatitis 2014;70:213-18.

21 Yazar K, Boman A, Liden C. Potent skin sensitizers in oxidative hair dye products on the Swedish market. Contact Dermatitis 2009;61:269-75. 


\section{Exposure assessment}

22 Yazar K, Boman A, Liden C. p-Phenylenediamine and other hair dye sensitizers in Spain. Contact Dermatitis 2012;66:27-32.

23 Sosted $\mathrm{H}$, Rustemeyer T, Goncalo $\mathrm{M}$, et al. Contact allergy to common ingredients in hair dyes. Contact Dermatitis 2013;69:32-9.

24 Gagliardi L, Ambroso M, Mavro J, et al. Exposure to paraphenylendiamine in hairdressing parlours. Int I Cosmet Sci 1992;14:19-31.

25 Hollund BE, Moen BE. Chemical exposure in hairdresser salons: effect of local exhaust ventilation. Ann Occup Hyg 1998;42:277-82.

26 Lind ML, Boman A, Sollenberg J, et al. Occupational dermal exposure to permanent hair dyes among hairdressers. Ann Occup Hyg 2005;49:473-80.

27 Lee HS, Lin YW. Permeation of hair dye ingredients, p-phenylenediamine and aminophenol isomers, through protective gloves. Ann Occup Hyg 2009;53: 289-96.

28 Lind ML, Johnsson S, Meding B, et al. Permeability of hair dye compounds p-phenylenediamine, toluene-2,5-diaminesulfate and resorcinol through protective gloves in hairdressing. Ann Occup Hyg 2007;51:479-85.

29 Gube M, Heinrich K, Dewes P, et al. Internal exposure of hairdressers to permanent hair dyes: a biomonitoring study using urinary aromatic diamines as biomarkers of exposure. Int Arch Occup Environ Health 2011;84:287-92.

30 Lind ML, Boman A, Surakka J, et al. A method for assessing occupational dermal exposure to permanent hair dyes. Ann Occup Hyg 2004;48:533-9.

31 Hueber-Becker F, Nohynek GJ, Meuling WJ, et al. Human systemic exposure to a [14C]-para-phenylenediamine-containing oxidative hair dye and correlation with in vitro percutaneous absorption in human or pig skin. Food Chem Toxicol 2004;42:1227-36.

32 Schettgen T, Heinrich $\mathrm{K}$, Kraus $\mathrm{T}$, et al. Determination of 2,5-toluylenediamine (2,5-TDA) and aromatic amines in urine after personal application of hair dyes: kinetics and doses. Arch Toxicol 2011;85:127-33.

33 Bregnhoj A, Menne T, Johansen JD, et al. Prevention of hand eczema among Danish hairdressing apprentices: an intervention study. Occup Environ Med 2012:69:310-16

34 Geens T, Dugardin S, Schockaert A, et al. Air exposure assessment of TDI and biological monitoring of TDA in urine in workers in polyurethane foam industry. Occup Environ Med 2012;69:93-8.

35 West BT, Welch KB, Galecki AT. Linear mixed models: a practical guide using statistical software. Taylor \& Francis Group, LCC, 2007.

36 Nixon R, Roberts $\mathrm{H}$, Frowen $\mathrm{K}$, et al. Knowledge of skin hazards and the use of gloves by Australian hairdressing students and practising hairdressers. Contact Dermatitis 2006;54:112-16.

37 Scientific Committee on Consumer Safety. Opinion on Toluene-2,5-diamine and its sulfate (COLIPA $n^{\circ}$ A5). Brussels: European Commission, Directorate-General for Health \& Consumers 2012:1-75.

38 Scientific Committee on Consumer Safety. Opinion on p-Phenylenediamine (COLIPA $n^{\circ}$ A7). Brussels: European Commission, Directorate-General for Health \& Consumers 2012:1-72.

39 Frasch HF, Fedorowicz A. Skin permeation calculator. NIOSH, 2012. 